\title{
Protée
}

\section{Le mouvement de la mémoire dans l’oeuvre de Nadia Myre}

\section{Marie Fraser}

Volume 32, numéro 1, printemps 2004

Mémoire et médiations

URI : https://id.erudit.org/iderudit/011023ar

DOI : https://doi.org/10.7202/011023ar

Aller au sommaire du numéro

Éditeur(s)

Département des arts et lettres - Université du Québec à Chicoutimi

ISSN

0300-3523 (imprimé)

1708-2307 (numérique)

Découvrir la revue

Citer cet article

Fraser, M. (2004). Le mouvement de la mémoire dans l'oeuvre de Nadia Myre. Protée, 32(1), 31-38. https://doi.org/10.7202/011023ar d'utilisation que vous pouvez consulter en ligne.

https://apropos.erudit.org/fr/usagers/politique-dutilisation/ 


\section{Nadia Myre}

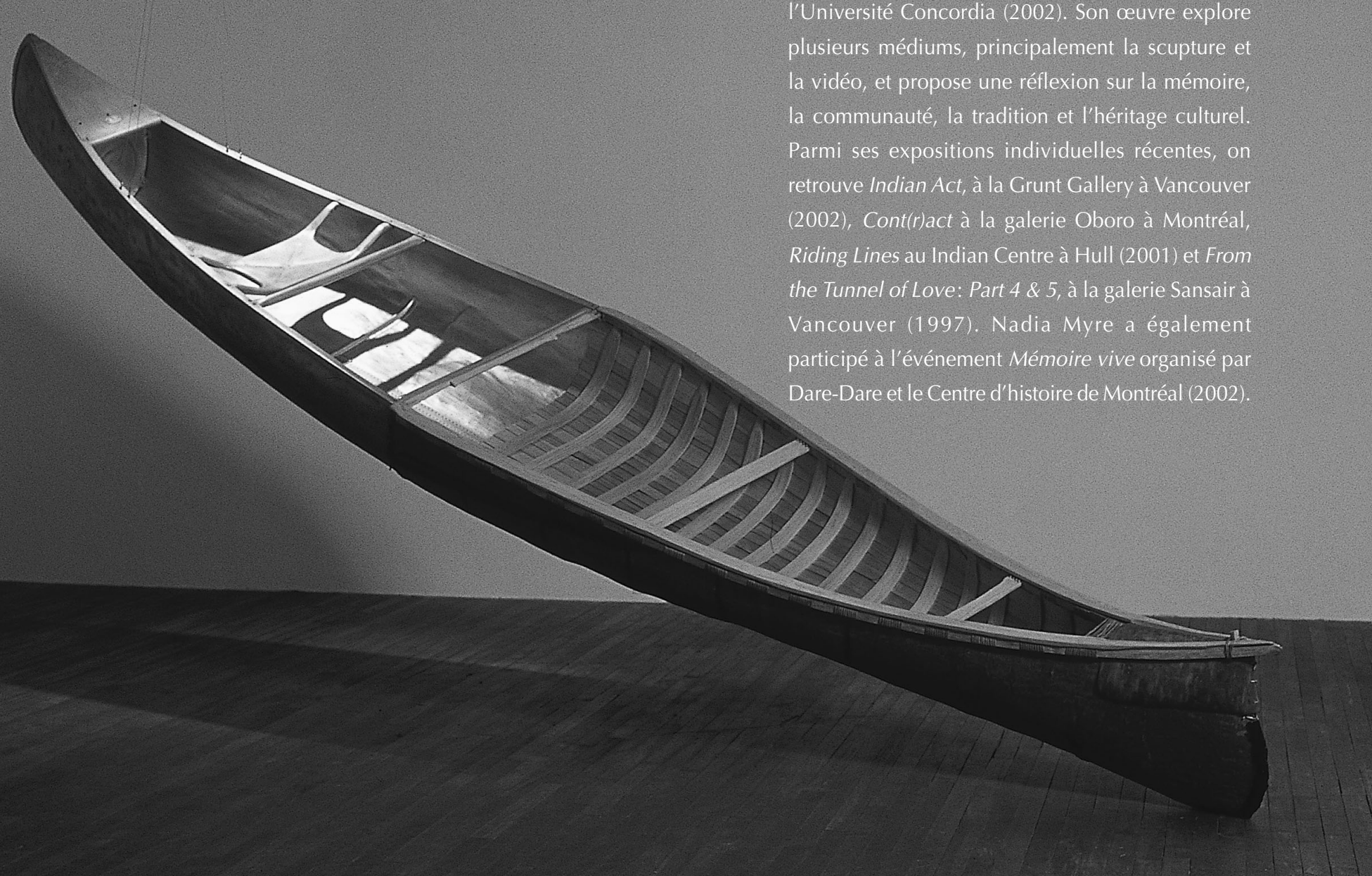
I'Emily Carr Institute of Art and Design (1997) et de IUniversité Concordia (2002). Son oeuvre explore plusieurs médiums, principalement la scupture et a el propose une refiexion sur la memoire communauté, la tradition et I'héritage culturel Parmi ses expositions individuelles récentes, on retrouve Indian Act, à la Grunt Gallery à Vancouver ding Lines au Indian Centre à Hiul the Tunnel of Love: Part 4 \& 5, à la galerie Sansair à nisé par

Née à Montréal, Nadia Myre est d’origine algonquine (Kitigan Zibi Anishnabeg), diplômée de 


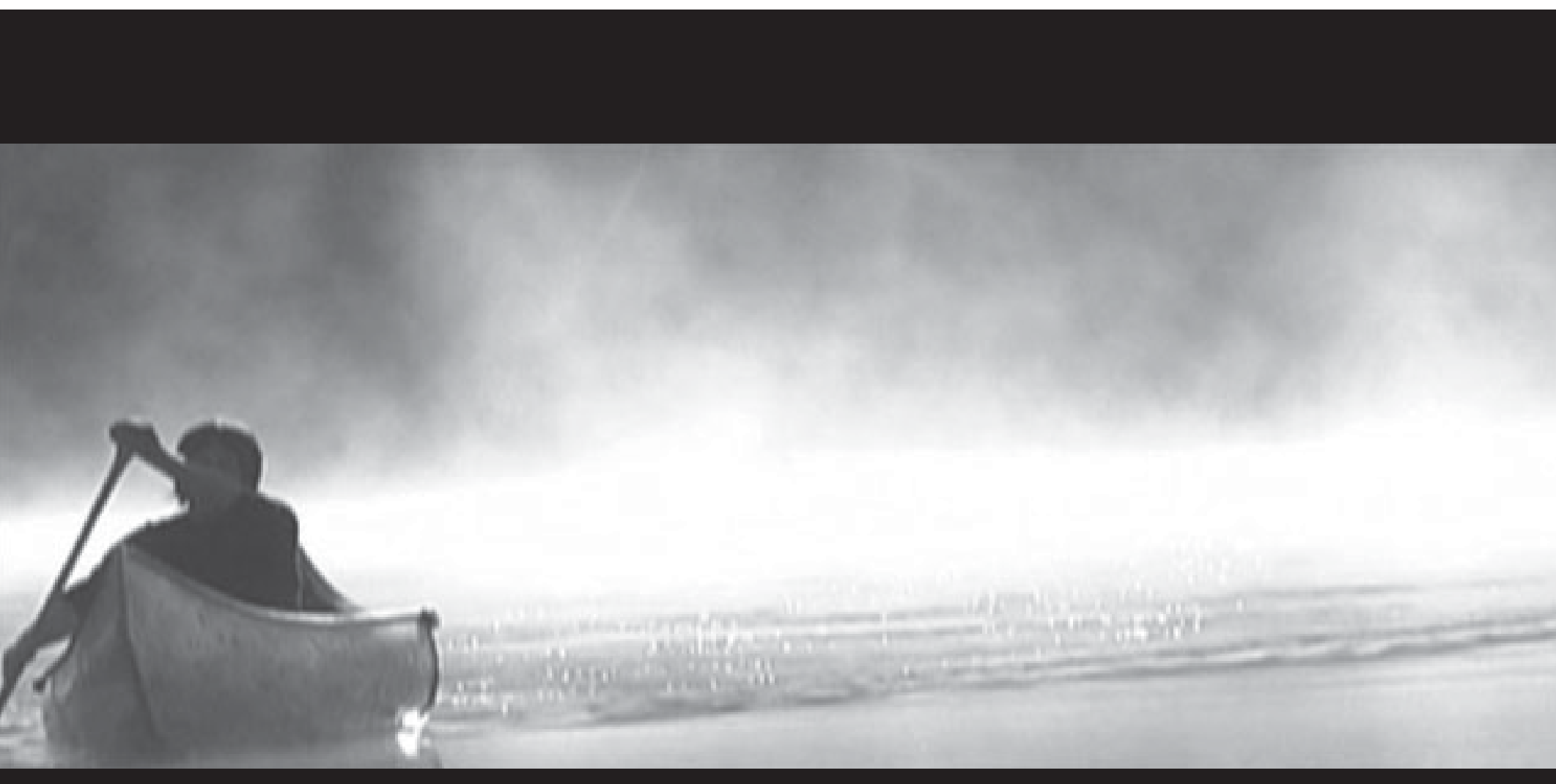

\footnotetext{
Portrait in Motion, 2001-2002. Film super-huit transféré sur bande-vidéo, 2 min 30 s (en boucle)

Caméra: Chang Wan Wee; caméra additionnelle: Marx Kruis; montage: Jack Hackel.
} 


\section{Le mouvement de la mémoire dans I'œuvre de Nadia Myre}

MARIE FRASER

Le court film intitulé Portrait in Motion (2001-2002) introduit de façon expérimentale et poétique le rôle particulier de la mémoire dans les oeuvres de Nadia Myre. Les deux minutes trente secondes pendant lesquelles un canoë s'approche lentement à la surface lisse et brumeuse d'un lac sont à peine suffisantes pour saisir le sens de ce mouvement. Au moment où la proximité de l'objet avec la caméra livre une image plus tangible, plus saisissable, le canoë effectue un virage et le film s'interrompt. L'attente est suspendue à ce portrait en mouvement pourtant fort simple mais insondable, et le film recommence sa boucle. Avant que l'image réapparaisse sur l'horizon à travers la brume, on entend d'abord des sons d'oiseaux, puis le bruit des déplacements sur l'eau.

Dans cette brève vidéo comme dans plusieurs de ses œuvres, Nadia Myre utilise des thèmes et des images «clichés» de ses origines pour transformer de telles représentations en une expérience purement visuelle et contemplative. L'atmosphère brumeuse de Portrait in Motion fait obstacle à la clarté et à I'objectivité, la mobilité du portrait défie son habituelle stabilité, et c'est ainsi que Nadia Myre fait basculer le mythe éthnographique de l'autochtone dans le récit d'une identité en quête d'elle-même et de sa reconnaissance. Portrait in Motion suggère le geste de réappropriation d'une mémoire, d'une identité et d'un imaginaire dont History in Two Parts (2001-2002) présente la part de dualisme en mettant en contact deux matérialités symbolisant la rencontre de deux réalités difficilement assimilables.

Indian Act (2001-2002) explore la même idée, mais, d'une poétique du regard, on assiste à un engagement plus directement politique. Au cours des trois dernières années, avec la participation de quelque deux cent trente personnes, Nadia Myre a progressivement brodé de petites perles de verre rouges et blanches sur chacune des cinquante-six pages des chapitres un à cinq de la Loi sur les Indiens. Associé à la vie domestique et à une technique traditionnelle, le travail du perlage détourne l'aspect légal et officiel du document. Porteur d'un paradoxe, cette réappropriation n'efface pas la loi ni ne conteste son existence, elle lui donne au contraire une matérialité plus abstraite, mais plus incisive, qui montre que la législation de l'identité et de l'appartenance est problématique. Indian Act réécrit le sens d'une histoire perdue à partir d'un geste qui cherche à conserver la mémoire vivante.

Ce projet interroge en quelque sorte l'espace clos et statique de l'écriture, en recouvrant un texte de loi grâce à un geste partagé collectivement par nombre d'individus, un geste qui s'inscrit dans la durée et dont les propriétés relationnelles ne sont pas sans lien avec l'oralité. The Distance Between Us (1997-2002) ainsi que Grandmother's Circle (2002) proposent aussi un tel rapprochement. Mais si ces dernières œuvres tentent de reconstruire symboliquement l'idée de communauté, d'espaces d'échange, de partage et de médiation, c'est afin d'en montrer la fragilité et la précarité. 


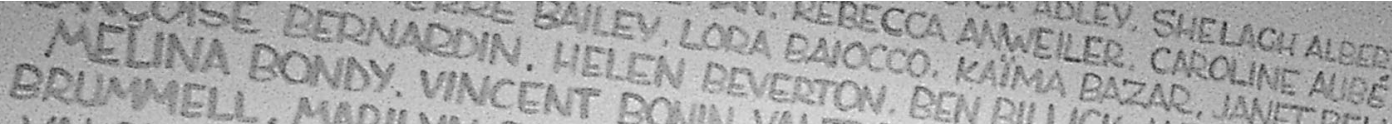

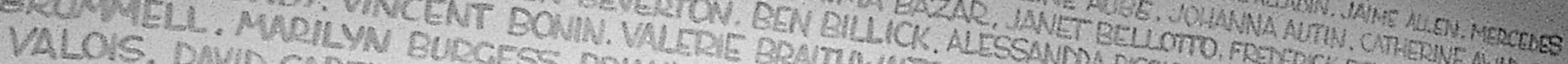

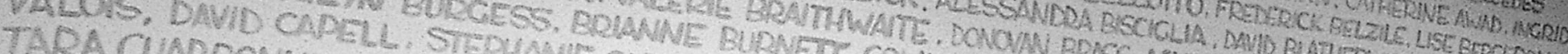

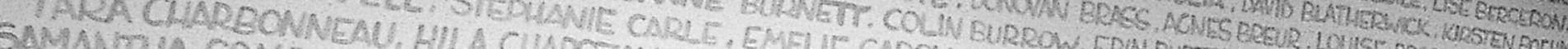

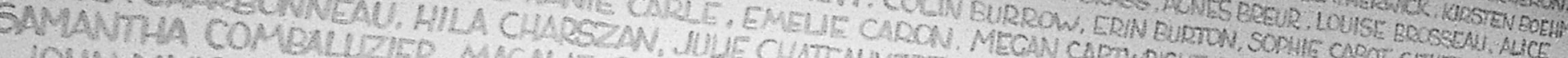

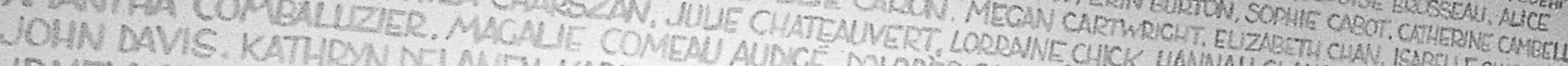

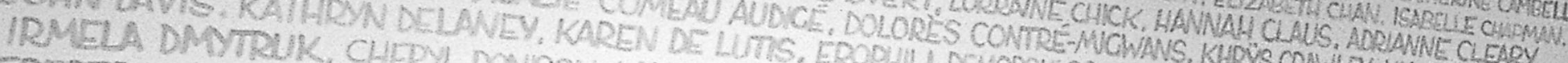

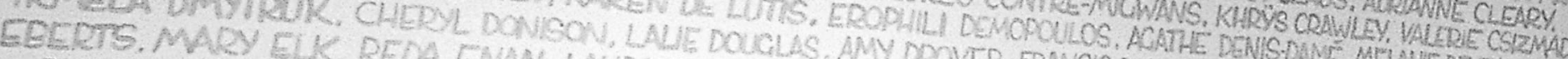

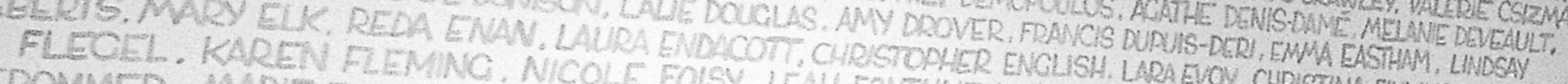

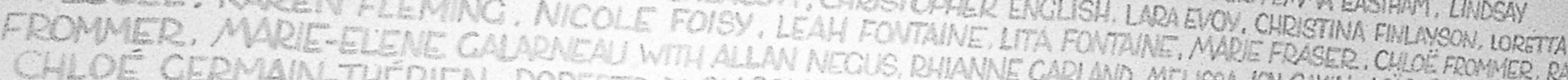

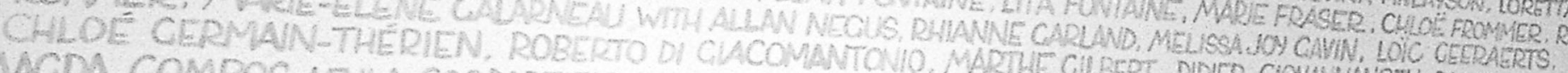

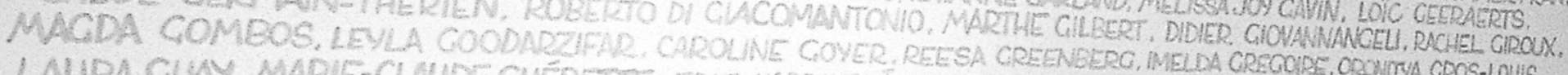

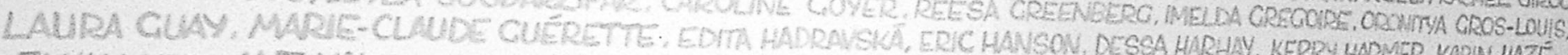

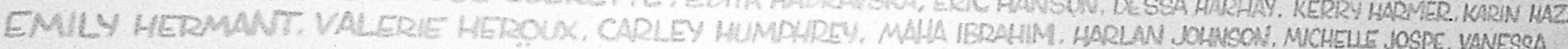

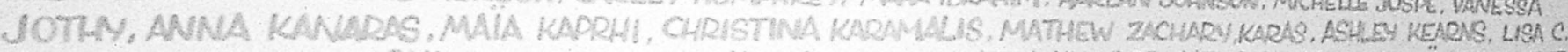

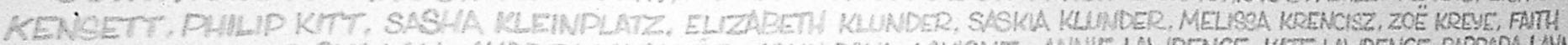
LA ROCOUE, YAT-CHI LAU, AUDPEY LAVALLEE. JOHN DAUL LAVICNE. ANUE LAWRENCE, KATE LAWRENCE, BARBARA LANE,

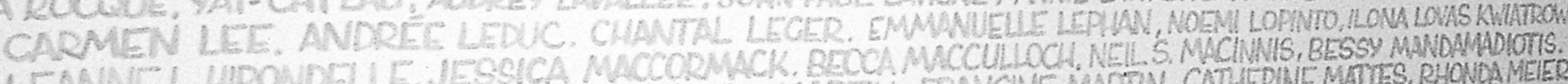

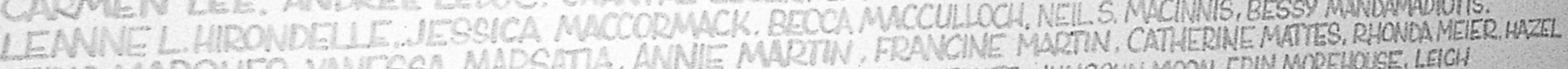

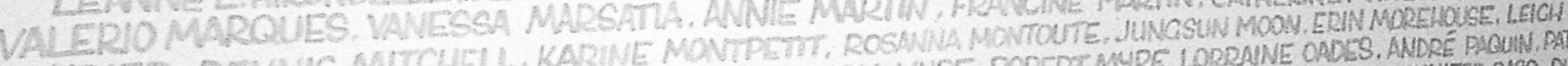

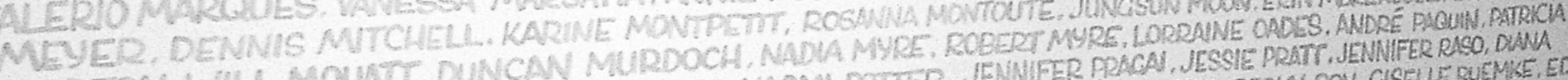

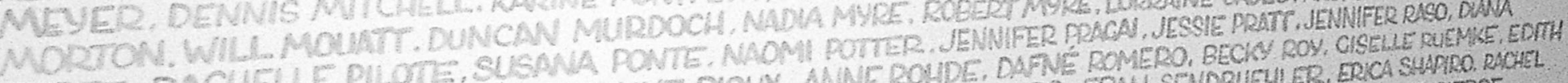

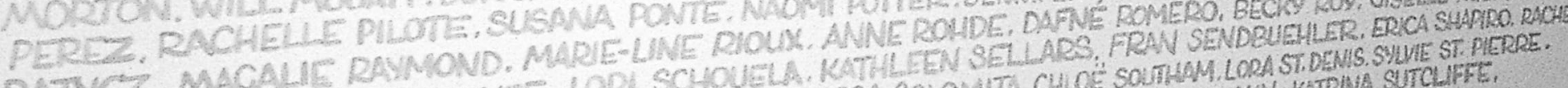

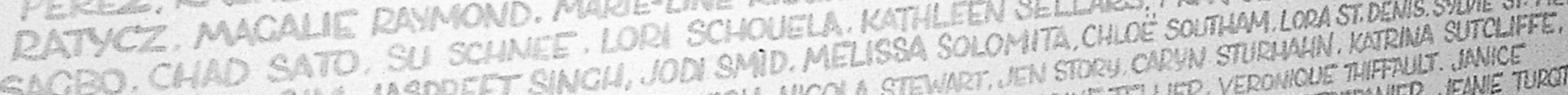

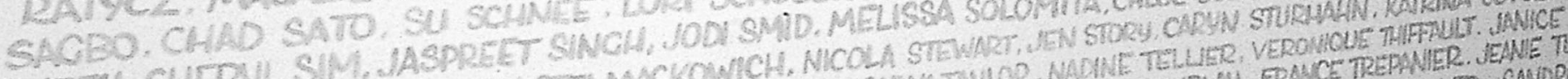

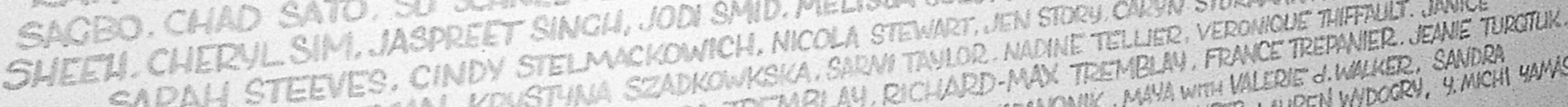

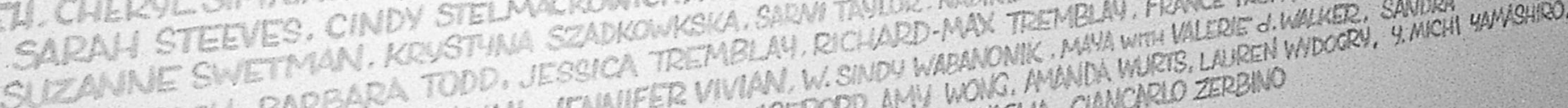

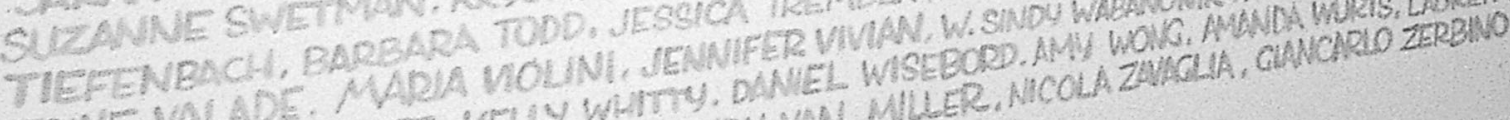

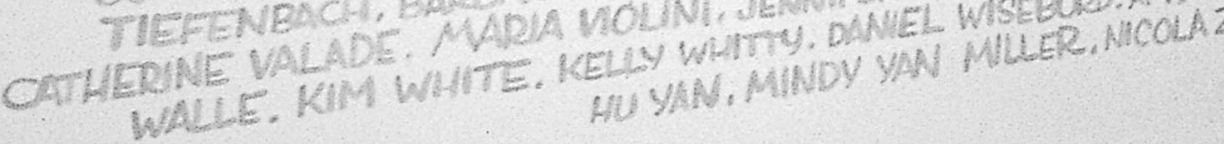

Noms des gens qui ont collaboré à Indian Act, 2001-2002. Indian Act est un long processus qui s'est échelonné sur plusieurs années. Rhonda Meier a élaboré et coordonné l'aspect collectif du projet. Photo: Denis Farley. 


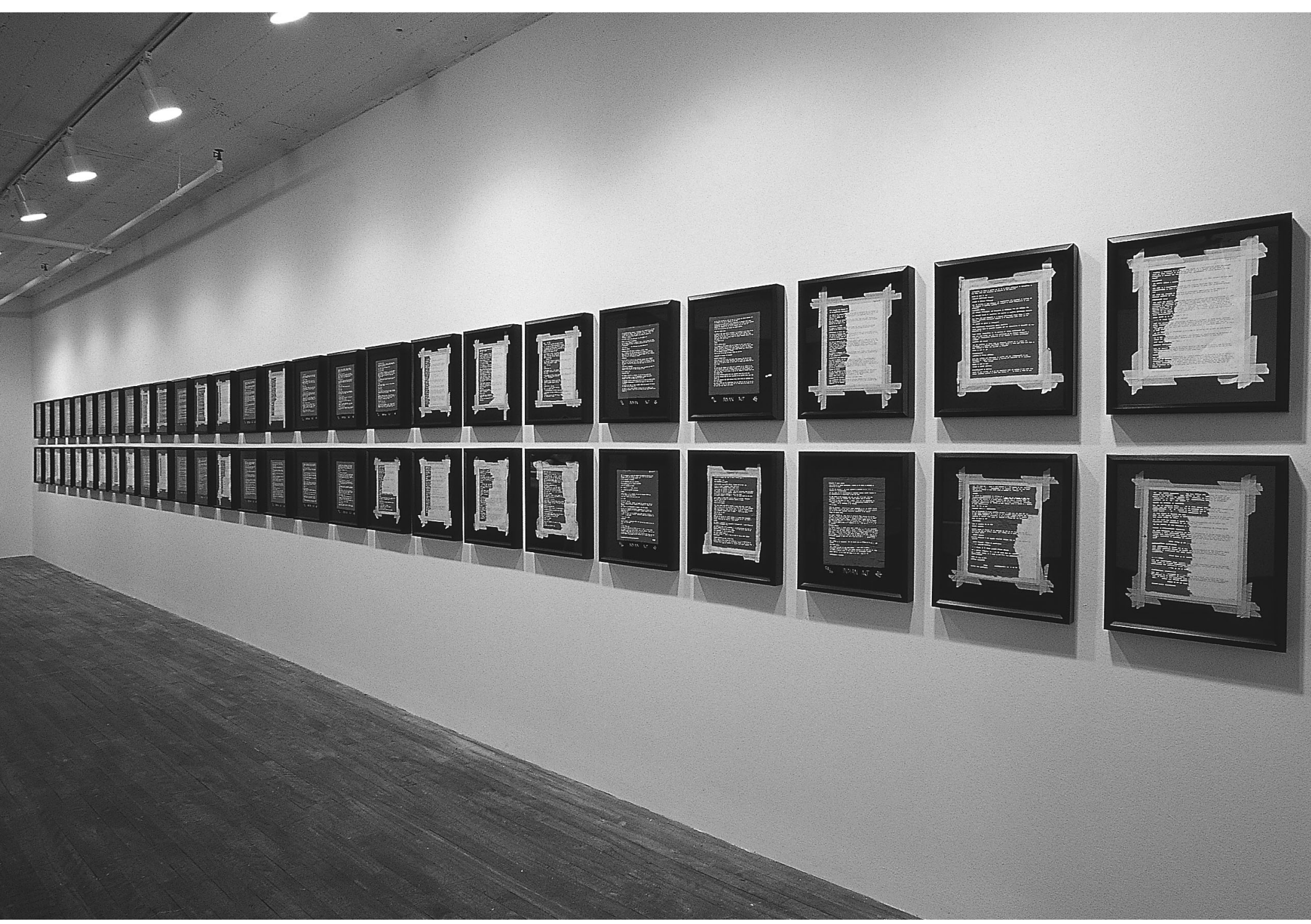

Indian Act, 2001-2002. Vue de l'installation lors de l'exposition Cont(r)act à la galerie Oboro, Montréal.

Perles de rocaille, feutre, fil. Texte de la Loi sur les Indiens, 1985, chap. 1-5, téléchargé et copié sur 56 pages. Photo: Denis Farley. 


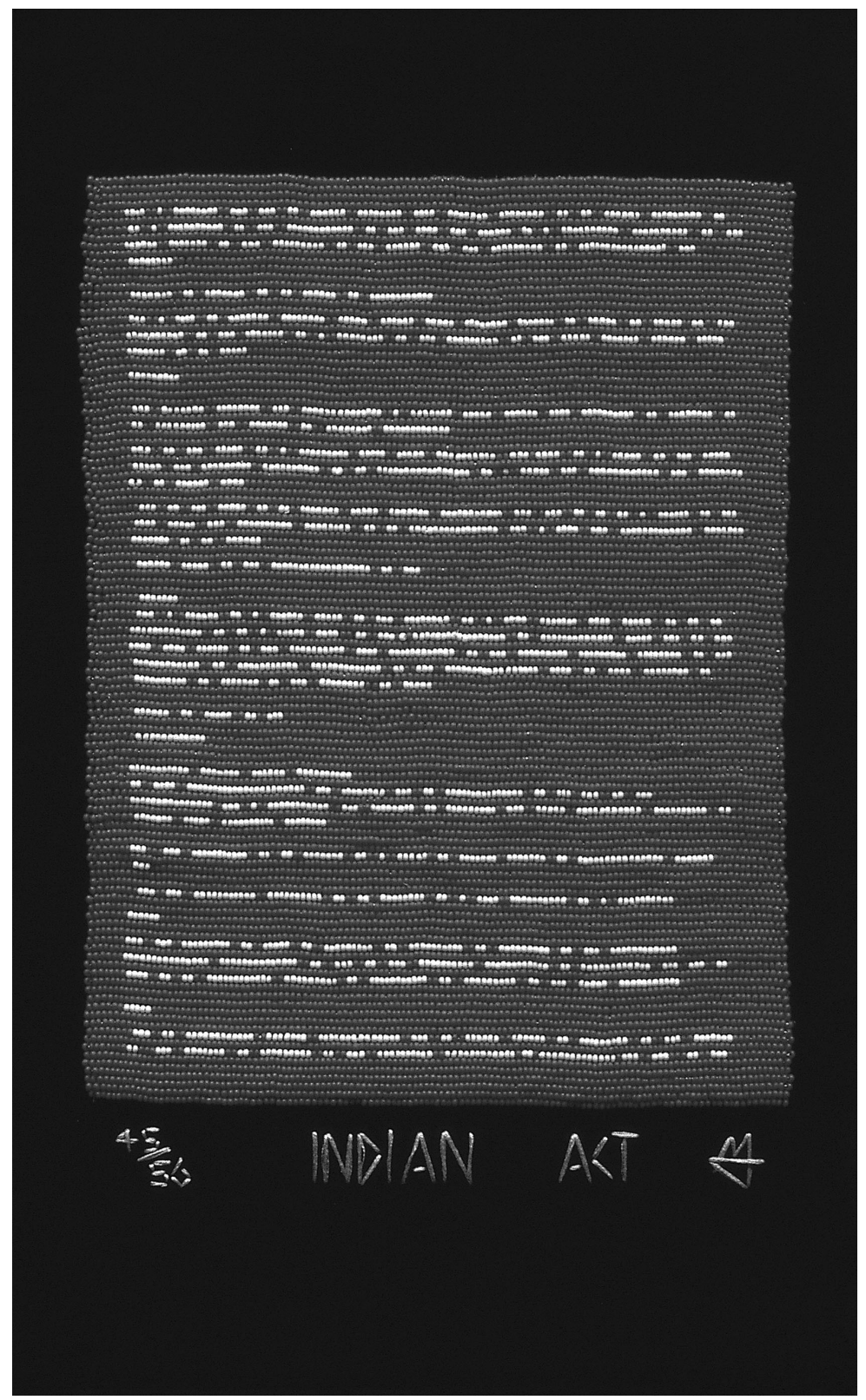

Indian Act, 2001-2002. Page 45, 46 × 38,5 cm chacune. Perlé par Barry Ace. Photo: Denis Farley. 


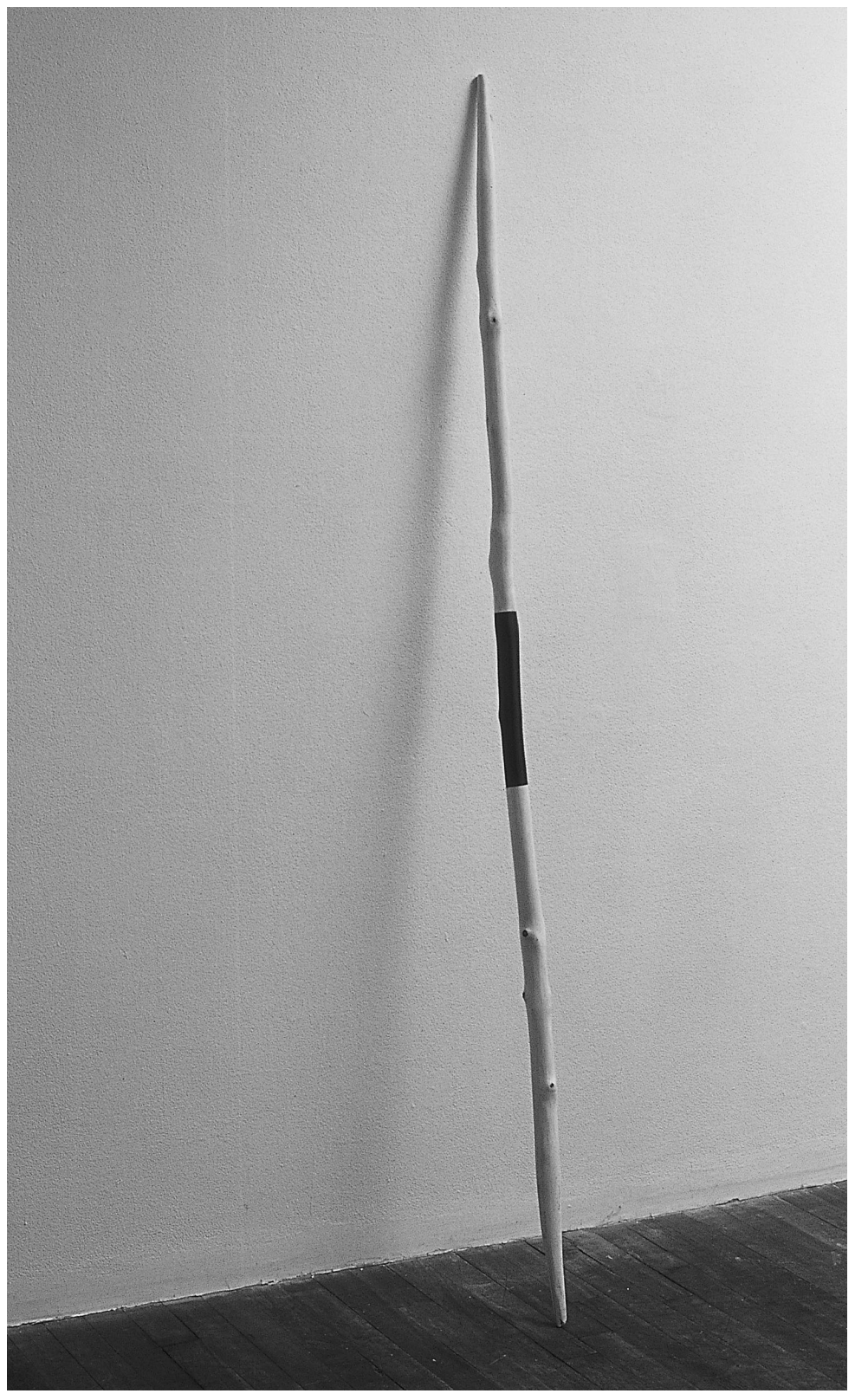

Grandmother's Circle, 2002. Bois, graphite. Photo: Denis Farley. 


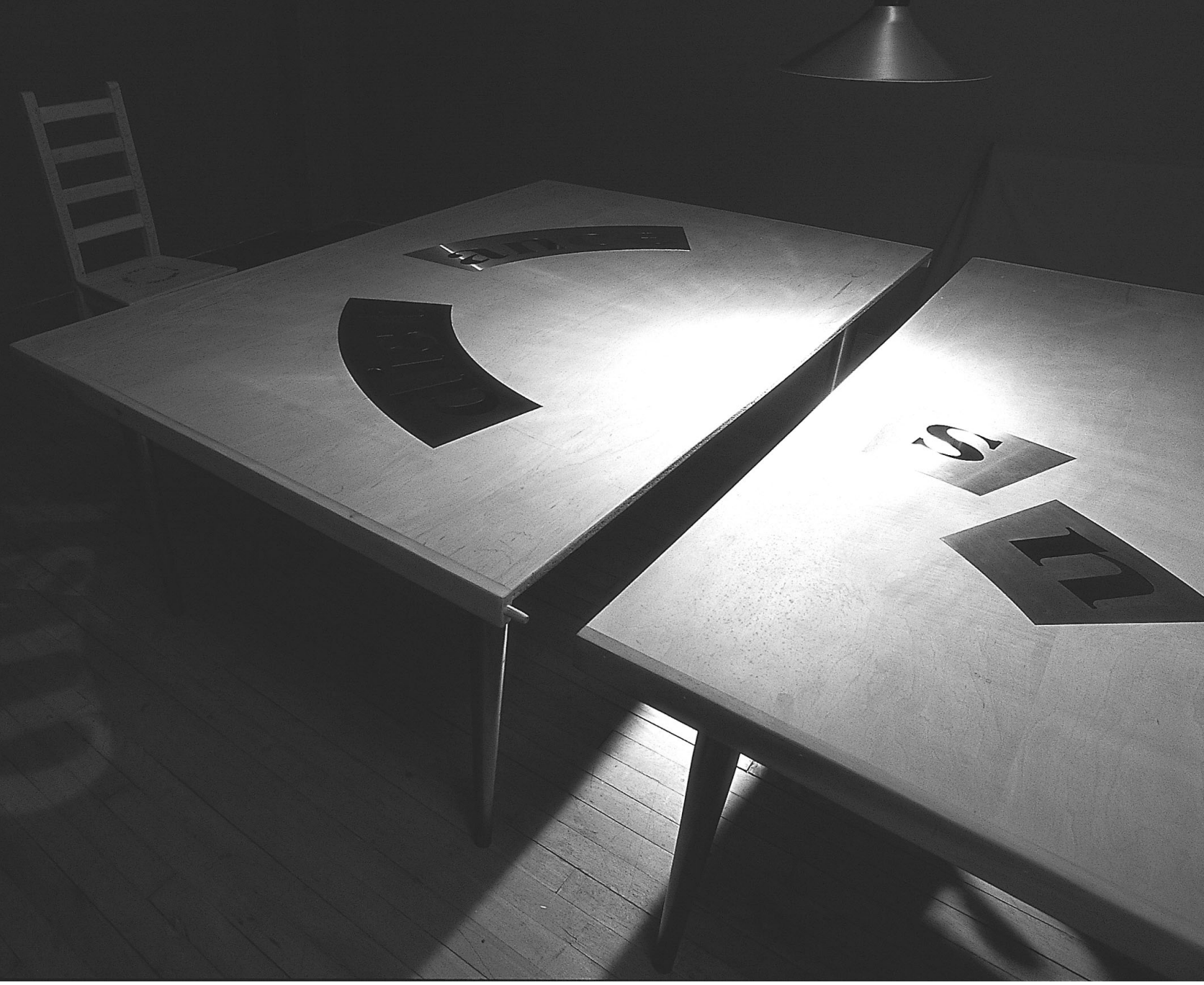

The Distance Between Us, 1997-2002. Installation technique mixte. Photo: Denis Farley. 